\title{
Atomic diffusion in metal-poor stars
}

\section{Predictions for the Spite plateau}

\author{
M. Salaris ${ }^{1,2}$ and A. Weiss ${ }^{2,3,4}$ \\ 1 Astrophysics Research Institute, Liverpool John Moores University, Twelve Quays House, Egerton Wharf, \\ Birkenhead CH41 1LD, UK \\ 2 Max-Planck-Institut für Astrophysik, Karl-Schwarzschild-Str. 1, 85748 Garching, Germany \\ 3 Institute for Advanced Study, Olden Lane, Princeton, USA \\ 4 Princeton University Observatory, Peyton Hall, Princeton, USA
}

Received 9 April 2001 / Accepted 5 July 2001

\begin{abstract}
We have computed a grid of up-to-date stellar evolutionary models including atomic diffusion, in order to study the evolution with time of the surface Li abundance in low-mass metal-poor stars. We discuss in detail the dependence of the surface Li evolution on the initial metallicity and stellar mass, and compare the abundances obtained from our models with the available Li measurements in Pop II stars. While it is widely accepted that the existence of the Spite Li-plateau for these stars is a strong evidence that diffusion is inhibited, we show that, when taking into account observational errors, uncertainties in the Li abundance determinations, in the $T_{\text {eff }}$ scale, and in particular the size of the observed samples of stars, the Spite plateau and the Li abundances in subgiant branch stars can be reproduced also by models including fully efficient diffusion, provided that the most metal-poor field halo objects are between 13.5 and 14 Gyr old. We provide the value of the minimum number of plateau stars to observe, for discriminating between efficient or inhibited diffusion. From our models with diffusion we derive that the average $\mathrm{Li}$ abundance along the Spite plateau is about a factor of 2 lower than the primordial one. As a consequence, the derived primordial $\mathrm{Li}$ abundance would be consistent with a high helium and low deuterium Big Bang Nucleosynthesis; this implies a high cosmological baryon density as inferred from the analyses of the cosmic microwave background.
\end{abstract}

Key words. stars: Population II - evolution - abundances - physical data and processes: diffusion

\section{Introduction}

Microscopic diffusion is a fundamental physical mechanism, which in principle has to be considered when computing stellar models. In particular, since the highly accurate seismic determination of the solar sound speed profile has become available, diffusion is an integral part of the standard solar model. Taking diffusion into account, not only the sound speed, but also the depth of the solar convective zone and the present surface helium abundance are in very good agreement with observations (see, for example Richard et al. 1996; Bahcall et al. 1997). Only in the region immediately below the convective envelope the theoretical models deviate from the seismic Sun significantly, indicating that diffusion might not operate exactly in the way calculated or pointing to some neglected additional physical process partially counter-working diffusion (Brun et al. 1999).

Send offprint requests to: M. Salaris, e-mail: ms@astro.livjm.ac.uk
With the Sun as our best laboratory for stellar physics strengthening the case for the presence of atomic diffusion, it immediately becomes mandatory to consider it also for other stars. Since diffusion is an intrinsically slow process operating over cosmic times, long-lived low-mass stars, in particular those of Population II, are evidently the best suited candidates.

Atomic diffusion affects the evolution of stars in two ways. In the deep interior, the sinking of helium towards the core leads to an effectively faster nuclear aging of the star, thus reducing its Main Sequence (MS) lifetime. For age determinations of globular clusters making use of the absolute brightness of the colour-magnitude diagram (CMD) turn-off (TO), when the cluster distance is known, diffusion eventually leads to lower ages; the effect accounts for a reduction of the order of $1 \mathrm{Gyr}$ for old globular clusters (Chaboyer et al. 1992; Castellani et al. 1997). The modified chemical structure also affects later evolutionary stages, such that age determination methods using differential quantities, for example the brightness 
difference between TO and horizontal branch, are less affected (about 0.5 Gyr; Cassisi et al. 1998; Castellani \& Degl'Innocenti 1999).

In the envelope of the star, diffusion leads to a depletion of the heavy elements and helium. This has the direct consequence that the present abundance of metals is lower than the initial one. In Salaris et al. (2000) we discussed in detail the consequences for the MS fitting method, the resulting distances, and subdwarfs ages. Furthermore, due to the decreased metals, the colours of the star change. The stellar radius and thus the effective temperature, however, also depend on the molecular weight in the star's core, which is increased in the presence of sedimentation. Taking diffusion into account, a much better agreement between models and observed colours or effective temperature results, as has been noted by Lebreton et al. (1999; see also Morel \& Baglin 1999 and Salaris et al. 2000). Similarly, an improved isochrone fitting for globular clusters, and consistency between the age from the cluster CMD and that from its integrated spectrum might be obtained (Vazdekis et al. 2001 for 47 Tuc).

A general prediction of models including atomic diffusion is that very metal-poor low-mass stars, with their high effective temperatures and thus very shallow convective envelopes, are readily depleted of metals over the MS lifetime (see the seminal paper by Deliyannis et al. 1990); this prediction can be contrasted with observations. In the present paper, we will therefore confront theoretical models with the observational result that the ${ }^{7} \mathrm{Li}$ abundance in such stars of $T_{\text {eff }} \gtrsim 5900 \mathrm{~K}$ forms a well-defined plateau below $[\mathrm{Fe} / \mathrm{H}] \approx-1.5$ (Spite \& Spite 1982). In the past, this so-called Li- or Spite-plateau has been used repeatedly as an, if not the argument against the full efficiency of diffusion (Deliyannis \& Demarque 1991; Vauclair \& Charbonnel 1998; Ryan et al. 1996), because the degree of depletion by diffusion increases with effective temperature. Stated differently, the necessity for an additional effect counteracting diffusion was claimed (Chaboyer et al. 1995a; Vauclair 1999). Candidate processes are rotationally induced mixing (Chaboyer et al. 1995a, 1995b) or a rather strong mass loss by a stellar wind (Vauclair \& Charbonnel 1995).

Although, therefore, the Li-plateau seems to make a strong case against diffusion, there are a number of issues that warrant a new look at this problem. First of all, the input physics for the stellar models has changed a lot in the last few years, and the improved equations of state, opacities and nuclear reaction rates all affect the stellar models (see Cassisi et al. 1998 for an overview). Secondly, and as a consequence, the deduced ages for the oldest clusters and stars has been reduced from 16-18 Gyr to 12-14 Gyr (Salaris et al. 1997). Thirdly, the observed field stars have a distribution of metallicities and possibly of ages, while often the comparison is performed by considering models of different masses all with the same age and metallicity (as, e.g., in Deliyannis et al. 1990 or Vauclair \& Charbonnel 1998). Finally, it is important to consider, when comparing theory with observations, not only the observational errors, but also the actual size of the sample of stars with Li abundance determinations.

The inclusion of diffusion does not only lead to a depletion of the surface lithium content, but to a second effect which could be measurable; the diffused $\mathrm{Li}$ is stored immediately below the thin convective envelope (see Vauclair \& Charbonnel 1998 for illustrative interior profiles) but remains at temperatures too low for lithium-destruction by proton capture. After the TO, the deepening convective envelope at first mixes this surplus to the surface, such that for some time subgiants should have Li-abundances somewhat above the Spite-plateau level which, in the case of effective diffusion, does not represent the primordial abundance. To recall, in case of no diffusion there is no difference between the initial Li-abundance and that on the main sequence and subgiant branch. With further expansion the convective envelope deepens until regions where the initial lithium was destroyed are diluting the envelope lithium content and a decrease in the surface abundance will ensue.

In this paper we will therefore investigate theoretical predictions for the Li-abundance ${ }^{1}$ of low-mass metal-poor stars, making use of the latest stellar models, which will be presented in Sect. 2. The calculations were used to prepare data about the lithium abundance as a function of metallicity, effective temperature and age. The comparison with the observed $\mathrm{Li}$ abundances of plateau and subgiant branch stars will be described in Sect. 3, while in Sect. 4 we will discuss the consequences of our results and, in particular, to which extent the Spite plateau can be used to make statements about the efficiency of diffusion operating in old metal-poor stars.

\section{Calculations and models}

We started out with stellar evolution calculations using the same input physics as in Salaris \& Weiss (1998) and Salaris et al. (2000). We computed $\alpha$ enhanced models (average enhancement by 0.4 dex, as in Salaris \& Weiss 1998) for initial iron contents $[\mathrm{Fe} / \mathrm{H}]_{\mathrm{o}}=-3.2,-2.6,-2.3,-1.8$ (corresponding to $Z=$ $0.000025,0.0001,0.0002,0.0006)$ and masses between 0.6 and $1.0 M_{\odot}$, from the pre-MS phase until the beginning of the Red Giant Branch (RGB). The initial He content has been fixed at $Y=0.23+3 Z$, and we used an initial lithium abundance $[\mathrm{Li}]_{\mathrm{o}}=2.50$. Diffusion was included by solving the Burgers equations following Thoul et al. (1994), and considered for H, He, Li, C, N, O, and Fe. The other elements have been assumed to diffuse like Fe. The rates for the $\mathrm{Li}$ destruction reactions are from the NACRE compilation (Angulo et al. 1999).

\footnotetext{
1 Throughout the paper we will indicate the abundance of $\mathrm{Li}$ with $[\mathrm{Li}]$, defined as $[\mathrm{Li}]=12+\log [N(\mathrm{Li}) / N(\mathrm{H})]$. We consider only ${ }^{7} \mathrm{Li}$ in our theoretical predictions for [Li], since the cosmological production of ${ }^{6} \mathrm{Li}$ is negligible in comparison with ${ }^{7} \mathrm{Li}$. Observations, however, determine the sum $\mathrm{Li}={ }^{7} \mathrm{Li}+{ }^{6} \mathrm{Li}$. We will come back to this point in the discussion at the end of the paper.
} 
Table 1. Surface lithium depletion (in dex) - due to nuclear burning only - along the pre-MS and MS, for various selected masses $\left(M / M_{\odot}\right)$ and initial metallicities $(Z)$. The four lines for each case correspond to the depletion after 10 and $100 \mathrm{Myr}, 1$ and 10 Gyr. For simplicity, we omit lines of higher age when the lithium abundance does not change any longer.

\begin{tabular}{l|ccc}
\hline$M / M_{\odot} \mid Z$ & $2.5 \times 10^{-5}$ & $2 \times 10^{-4}$ & $6 \times 10^{-4}$ \\
\hline 0.60 & -0.75 & -0.63 & -0.42 \\
& -1.03 & -0.90 & -0.79 \\
& -1.36 & -0.96 & -0.89 \\
& -1.83 & -1.83 & -1.76 \\
0.65 & -0.19 & -0.20 & -0.19 \\
& -0.19 & -0.21 & -0.23 \\
& -0.19 & -0.22 & -0.25 \\
& -0.19 & -0.22 & -0.26 \\
0.70 & -0.03 & -0.06 & -0.07 \\
& -0.04 & -0.06 & -0.07 \\
& -0.04 & -0.06 & -0.08 \\
0.75 & -0.01 & -0.02 & -0.03 \\
0.80 & -0.00 & -0.01 & -0.01 \\
\hline
\end{tabular}

Pre-MS evolution was followed to assess the possible Li-depletion in this phase, although it is expected to lead to substantial lithium destruction only for stellar masses below $0.8 M_{\odot}$ (D'Antona \& Mazzitelli 1984; Deliyannis et al. 1990). For these pre-MS calculations, the Garching stellar evolution code was used (see, e.g., Weiss \& Schlattl 2000 and references therein), in contrast to the variant of the FRANEC program for all other calculations. Both codes use in most aspects essentially the same and in some (opacities, diffusion coefficients) identical physics and produce very similar results, as shown, for example, in Weiss \& Schlattl (2000). The derived Li abundances at the Zero Age Main Sequence where then provided to the code we have employed in all our previous evolutionary computations. Selected results about the surface Li-depletion exclusively due to nuclear burning are summarized in Table 1.

These numbers can be compared with, for example, the results of Deliyannis et al. (1990). We get a definitely higher depletion at the lower masses than in these older calculations (with older opacities and equation of state). For example (see their Fig. 14), our $0.60 M_{\odot}$ model shows strong depletion already on the pre-MS for all metallicities, and continuing depletion on the MS, while the depletion in the $Z=0.0001$ models by Deliyannis et al. (1990) levels off at -0.4 dex already at the beginning of the MS phase. This behaviour is similar to that found by Ventura et al. (1998), when comparing with their older models (D'Antona \& Mazzitelli 1984): the more up-todate physics leads to a higher depletion.

When comparing our diffusion calculations with the results from the scaled-solar models by Vauclair \& Charbonnel (1998), for ages of 12 and 14 Gyr, we find
Table 2. Lithium depletion, given as $\mathrm{Li}(t) / \mathrm{Li}_{0}$, in models with diffusion. The third column shows results of Vauclair \& Charbonnel (1998) for $[\mathrm{Fe} / \mathrm{H}]=-2$ ("VC"), the fourth one those of the present paper at the same $[\mathrm{Fe} / \mathrm{H}]$, and the last one at the same total metallicity, $[\mathrm{M} / \mathrm{H}]$. The comparison is done for three masses (Col. 1) in common and two ages, 12 and 14 Gyr (Col. 2).

\begin{tabular}{l|l|ccc}
\hline$M / M_{\odot}$ & $t$ & $\mathrm{VC}$ & {$[\mathrm{Fe} / \mathrm{H}]=-2$.} & {$[\mathrm{M} / \mathrm{H}]=-2$.} \\
\hline 0.65 & 12 & 0.700 & 0.431 & 0.436 \\
& 14 & 0.662 & 0.412 & 0.427 \\
0.70 & 12 & 0.652 & 0.695 & 0.667 \\
& 14 & 0.595 & 0.657 & 0.622 \\
0.75 & 12 & 0.465 & 0.482 & 0.398 \\
& 14 & 0.384 & 0.365 & 0.245 \\
\hline
\end{tabular}

good agreement, with large differences only for the $M=$ $0.65 M_{\odot}$ models, which are mainly due to differences in the pre-MS phase, since for these masses and ages the depletion due to diffusion from the convective envelope is only a small contribution.

From the computed grid of stellar models we then produced Li-isochrones (i.e. Li-abundances along isochrones as in Deliyannis et al. 1990) for different ages and different initial $[\mathrm{Fe} / \mathrm{H}]$ values. It is important to remark here that along an isochrone the surface $[\mathrm{Fe} / \mathrm{H}]$ is not constant, again due to the effect of diffusion (Morel \& Baglin 1999; Salaris et al. 2000). At a given age of some Gyr, the surface $[\mathrm{Fe} / \mathrm{H}]$ is lower than the initial value; it also shows a decrease towards the TO and than increases along the Sub Giant Branch (SGB) until it reaches the initial value along the RGB (see Fig. 2). Therefore our isochrones, for ages larger than $\sim 5 \mathrm{Gyr}$, cover a range of surface $[\mathrm{Fe} / \mathrm{H}]$ between $\sim-2.0$ and $\sim-3.8$.

Figure 1 displays in panels a) and b) Li-isochrones as a function of $T_{\text {eff }}$ and mass for ages of 12, 13, 14 Gyr and initial metallicity $[\mathrm{Fe} / \mathrm{H}]_{\mathrm{o}}=-2.6$, from the unevolved lower main sequence up to the TO. The shape of the isochrones looks very similar for the various ages apart from the different value of $T_{\text {eff }}$ at both the TO and at the cool end. $\mathrm{Li}$ depletion at the coolest end of the isochrones is dominated by the pre-MS depletion (masses populating these region range from 0.60 up to $\sim 0.65 M_{\odot}$ ), while for $T_{\text {eff }}$ larger than $\sim 5800 \mathrm{~K} \mathrm{Li}$ diffusion is mainly responsible for the surface Li depletion, since pre-MS depletion is negligible. The isochrones for $t=12$ Gyr show more depletion at the TO since, in spite of the shorter time available for diffusion to deplete the surface Li, TO stars are hotter and have shallower convective regions and therefore the surface Li depletion is more efficient. The Li-isochrones show also an extended region, with $T_{\text {eff }}$ ranging between $\sim 5800$ and $6300 \mathrm{~K}$ (the extension depends on the age), in which the depletion does not change by more than 0.2 dex. At these ages the depletion at the level of this plateau is of the order of 0.3 dex. 


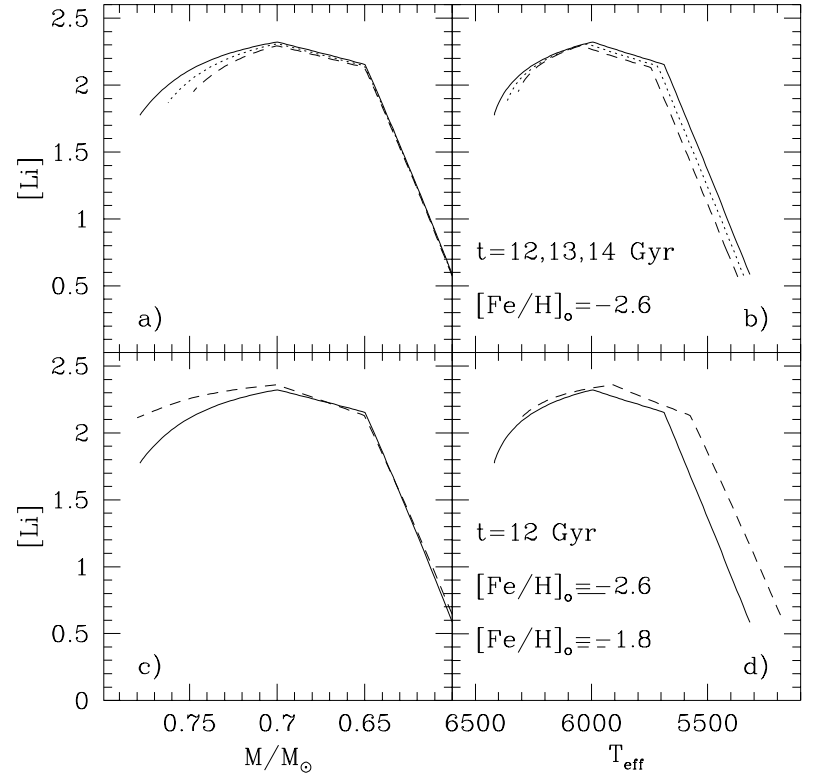

Fig. 1. Panels a) and $\mathbf{b}$ ): Run of [Li] as a function of the stellar mass and $T_{\text {eff }}$ for isochrones with initial metallicity $[\mathrm{Fe} / \mathrm{H}]_{\mathrm{o}}=$ -2.6 and ages of 12 (solid line), 13 (dotted line) and $14 \mathrm{Gyr}$ (dashed line). Panels c) and d): As in panels a) and b), but for isochrones with $t=12 \mathrm{Gyr}$ and $[\mathrm{Fe} / \mathrm{H}]_{\mathrm{o}}=-2.6$ (solid line) and -1.8 (dashed line).

In panels c) and d) of Fig. 1 we compare the [Li] values along two isochrones with the same age of 12 Gyr but two different initial metallicities. The shape of the isochrones again is very similar, with the more metal-rich models being less depleted at the TO (larger convective envelopes) and in general more Li-rich for a given value of $T_{\text {eff }}$.

Finally, in Fig. 2 we display the run of [Li] as a function of $T_{\text {eff }}$ for 12 and 14 Gyr isochrones with $[\mathrm{Fe} / \mathrm{H}]_{\mathrm{o}}=-2.6$, from the MS to the base of the RGB. It is very interesting to notice that after the TO $[\mathrm{Li}]$ continues to decrease for the next $50 \mathrm{~K}$, with a further depletion by about 0.1 dex. The maximum depletion is a function of age, strongly decreasing for increasing age. It also depends on metallicity, in the sense that at a given age the maximum depletion decreases with increasing metallicities. This behaviour is easy to understand if one recalls that the extension of the convective envelope in TO stars increases for increasing age at a given metallicity, or for increasing metallicity at a given age, reducing thus the effect of diffusion on the surface abundances.

Later on, with decreasing temperature, the [Li] abundance starts to rise again due to the deepening of the convective region which returns to the surface the Li diffused outside the envelope along the MS. [Li] reaches a peak when $T_{\text {eff }} \simeq 5600-5700 \mathrm{~K}$, with an abundance within 0.1 dex from the initial one; then the Li surface abundance starts to drop, since the convective envelope reaches regions where Li had been burned due to the higher temperatures of the stellar matter. We note that the location and level of the Li abundance peak is fairly independent of age and metallicity (see Fig. 7) and, in addition, is within

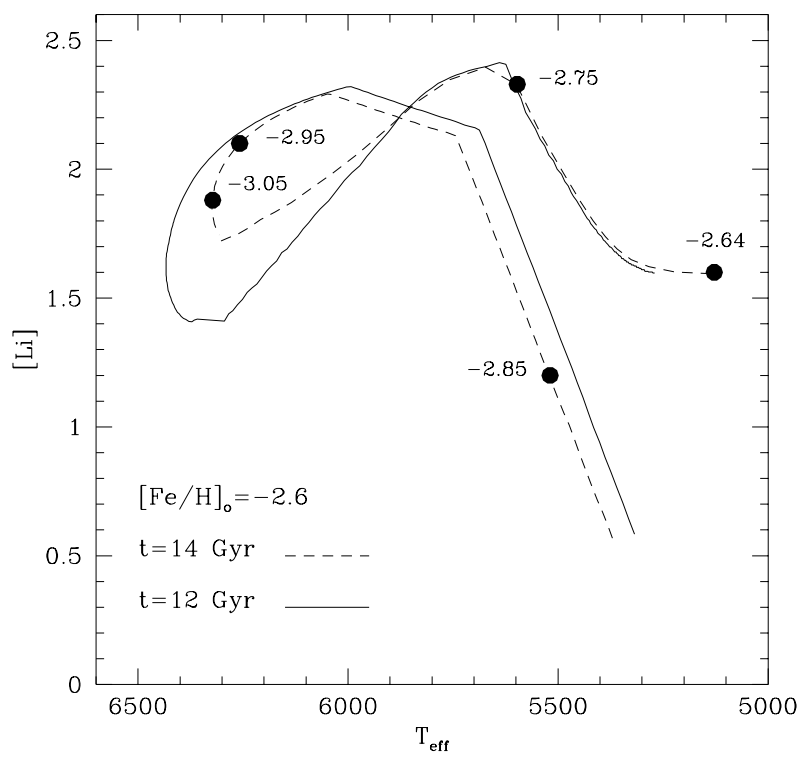

Fig. 2. [Li] as a function of $T_{\text {eff }}$ for isochrones of 12 and $14 \mathrm{Gyr}$, with $[\mathrm{Fe} / \mathrm{H}]_{\mathrm{o}}=-2.6$, from the MS to the lower RGB. Selected values of $[\mathrm{Fe} / \mathrm{H}]$ along the $14 \mathrm{Gyr}$ isochrone are also shown.

0.1 dex of the primordial value, independent of the effectiveness of diffusion. If this peak is found by analyzing the [Li] distribution in a large sample of stars spanning the entire SGB temperature range, it would provide an estimate of the primordial $\mathrm{Li}$ abundance to this accuracy.

\section{Comparison with observations}

In this section we will compare the behaviour of the [Li] abundances obtained from our MS and SGB models with the observations of metal-poor field and globular cluster stars. We concentrate our attention on stars with $[\mathrm{Fe} / \mathrm{H}] \leq-2.0$, which constitute the largest fraction of the currently available samples of PopII Li-plateau stars; in the last part of this section we will discuss briefly also the case of stars with $[\mathrm{Fe} / \mathrm{H}]$ up to $\sim-1.5$, which is approximately the upper metallicity boundary of the Spite plateau.

\subsection{Field MS stars}

Observations of Li abundances in metal-poor MS field stars provide a qualitative picture that is basically consistent among the different authors, but which can differ a lot in the details (see, e.g., Thorburn 1994; Ryan et al. 1996; Spite et al. 1996; Bonifacio \& Molaro 1997; Ryan et al. 1999; Ryan et al. 2001 and references therein). The main result (as an example we show in Fig. 3 the Thorburn 1994 data for the metallicity range spanned by our models) is that metal-poor MS stars with $T_{\text {eff }}$ larger than approximately $5800 \mathrm{~K}$ show a remarkably constant [Li] value (Li-plateau), while there is a larger depletion at lower temperatures, increasing for decreasing temperature. Moreover, in the plateau region, a handful of stars 

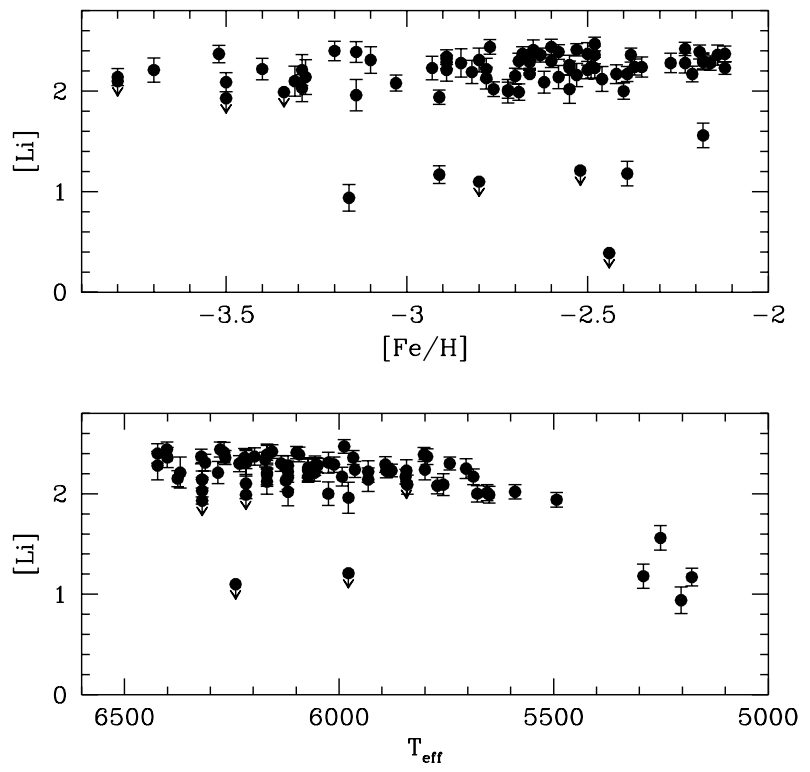

Fig. 3. Run of $[\mathrm{Li}]$ as a function of $[\mathrm{Fe} / \mathrm{H}]$ (upper panel) and $T_{\text {eff }}$ (lower panel) from the Thorburn (1994) data for MS stars with $[\mathrm{Fe} / \mathrm{H}] \leq-2.0$. Upper limit detections are also shown. For the sake of clarity we did not plot the error bars on the $T_{\text {eff }}$ values (of the order of, typically, $80-100 \mathrm{~K}$, see Thorburn 1994 for more details).

show a much lower [Li] than the plateau counterpart (e.g. Thorburn 1994).

The details of this picture, however, differ from author to author. The exact $T_{\text {eff }}$ location and extent of the plateau varies slightly; some trend of [Li] with $T_{\text {eff }}$ along the plateau was detected by Thorburn (1994) and Ryan et al. (1996), but it is absent in the analyses by Bonifacio \& Molaro (1997) and Ryan et al. (1999). A trend of [Li] with respect to $[\mathrm{Fe} / \mathrm{H}]$ for plateau stars is claimed by Thorburn (1994), Ryan et al. (1996), Ryan et al. (1999) and Ryan et al. (2001), while it is missing in Bonifacio \& Molaro (1997). Also the absolute average value of [Li] for plateau stars shows differences between different authors.

In addition to the purely observational errors and differences in the adopted metallicity scale, there are other sources of uncertainty that should be mentioned. The first one is the set of model atmospheres used in the spectral analyses. As dicussed by Ryan et al. (1996), the differences between $\mathrm{Li}$ abundances derived from different sets of model atmospheres show trends with respect to both $T_{\text {eff }}$ and $[\mathrm{Fe} / \mathrm{H}]$. Very recently Asplund et al. (1999) discussed the differences in the $[\mathrm{Li}]$ values derived using either 1D, plane parallel, hydrostatic model atmospheres, like the MARCS (Gustafsson et al. 1975 with subsequent updates) and ATLAS9 (Kurucz 1993) ones used in all recent papers on $[\mathrm{Li}]$ abundances, or their 3D hydrodynamical model atmospheres. A remarkable result is that not only the absolute values of [Li] are changed when passing from $1 \mathrm{D}$ to $3 \mathrm{D}$ models, but that also the spread in $[\mathrm{Li}]$ values is changed. They discuss the case of HD 140283 and HD 84937 whose [Li] derived from MARCS models is, respectively, 2.12 and 2.28. Using their 3D hydrodynamical models, Asplund et al. (1999) obtain 1.78 and 2.08; the difference between the two values is almost doubled with respect to the case of $1 \mathrm{D}$ hydrostatic models. Keeping in mind their cautionary remarks about the assumption of LTE in their models, it is nevertheless important to notice that the derived [Li] spread may underestimate the real variation.

Another important source of uncertainty is the $T_{\text {eff }}$ scale itself. Different authors use temperature scales which often differ not just by a constant offset, but also show differential trends with respect to the metallicity. To investigate better this point we have considered 8 plateau stars in common among the investigations by Thorburn (1994), Ryan et al. (1999) and Ryan et al. (1999).

It turned out that the spread of the $T_{\text {eff }}$ values is not constant and in some cases quite remarkable. The differences of the Ryan et al. (1999) $T_{\text {eff }}$ values to the Bonifacio \& Molaro (1997) ones range from about $-150 \mathrm{~K}$ up to almost $300 \mathrm{~K}$. Somewhat smaller values (ranging between about $-20 \mathrm{~K}$ and $150 \mathrm{~K}$ ) are obtained when considering the difference to the $T_{\text {eff }}$ by Thorburn (1994). If one takes into account that a $T_{\text {eff }}$ variation by $100 \mathrm{~K}$ changes the derived [Li] value by about 0.07 dex (Ryan et al. 1999), it may well be that the current uncertainty about Li trends along the plateau is due mainly to uncertainties of the temperature scale. Also the $[\mathrm{Fe} / \mathrm{H}]$ values adopted in different investigations show a spread which reaches values larger than \pm 0.5 dex.

In order to analyze the predictions of theoretical diffusive models regarding the trends of $[\mathrm{Li}]$ with respect to $T_{\text {eff }}$ and $[\mathrm{Fe} / \mathrm{H}]$ in metal-poor stars, we resorted to MonteCarlo (MC) simulations using our own isochrones. In this way we will be able to take into account the fact that the subdwarfs with $[\mathrm{Li}]$ measurements have a metallicity distribution and possibly an age distribution. As we have shown before, the initial metallicity and age alter the location and shape of $\mathrm{Li}$-isochrones, and it is important to take these two factors into account. Moreover, with MC simulations we will be also able to account for the actual number of MS stars with [Li] measurements. This point as we will see later on - is of fundamental importance when trying to constrain the efficiency of diffusion from Li-plateau stars. It is important to remark at this stage that, since the observations provide contradictory results about the properties of the Spite plateau, we can just discuss whether our models with diffusion are able to reproduce the plateau, and if its derived properties are within the range allowed by existing data.

In our simulations we assumed that present [Li] observations of field MS subdwarfs cover also TO stars. We prescribed an age-initial metallicity relationship and, by interpolating among our isochrones, we have drawn a large sample of $10000 \mathrm{MS}$ stars, according to a Salpeter Initial Mass Function, with an initial $[\mathrm{Fe} / \mathrm{H}]$ distribution adjusted (by trial and error) in such a way that the 10000 stars show a metallicity distribution in agreement with the typical metallicity distribution of halo stars as determined by Ryan \& Norris (1991). For each star we determined the 

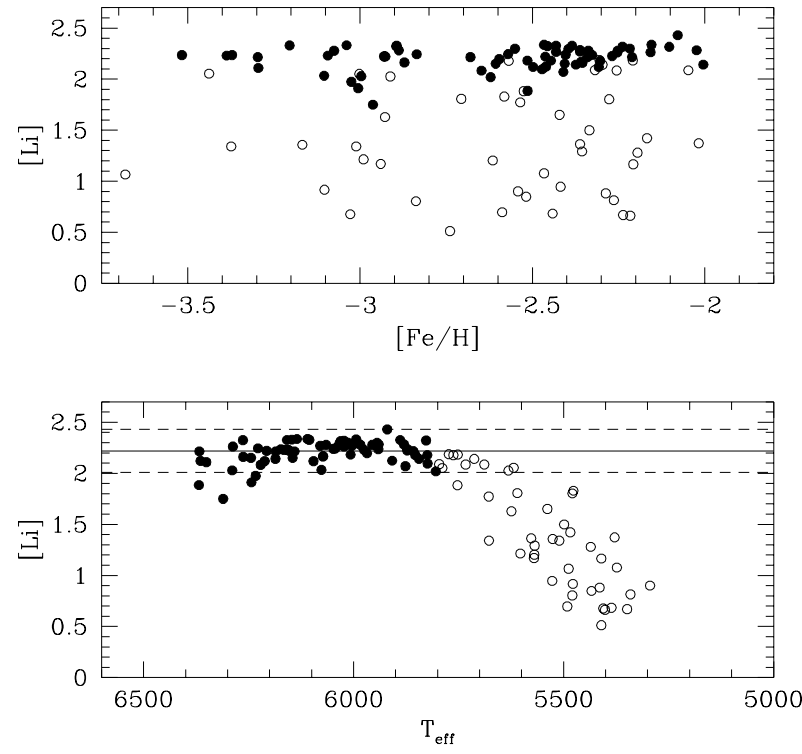

Fig. 4. Run of [Li] as a function of $[\mathrm{Fe} / \mathrm{H}]$ (upper panel) and $T_{\text {eff }}$ (lower panel) for stars in one of our MC simulations, considering an age randomly distributed between 13.5 and 14 Gyr. Filled circles represent plateau stars (see text for details).

value of its mass, bolometric luminosity, surface $[\mathrm{Fe} / \mathrm{H}]$ and $[\mathrm{Li}]$, and $T_{\text {eff. }}$. We then added to the values of $[\mathrm{Fe} / \mathrm{H}]$, [Li], and $T_{\text {eff }} 1 \sigma$ random observational errors by, respectively, 0.20 dex, 0.07 dex and $65 \mathrm{~K}$. These observational errors are approximately an average of the values quoted by Thorburn (1994), Ryan et al. (1996), Spite et al. (1996), Bonifacio \& Molaro (1997).

From this sample of 10000 stars representing the halo population we have randomly drawn 30 smaller samples of 120 stars each, which represent the MC realizations of the observational data; about 65 objects populate the plateau region (which we define as being the region with $T_{\text {eff }}>5800 \mathrm{~K}$ ). This number of plateau stars correponds approximately to the largest sample with [Li] abundances and $[\mathrm{Fe} / \mathrm{H}] \leq-2.0$ available to this day (see, e.g., Thorburn 1994; Ryan et al. 1996). For each of these samples we studied the distribution of stars in the $[\mathrm{Li}]-T_{\text {eff }}$ and $[\mathrm{Li}]-[\mathrm{Fe} / \mathrm{H}]$ planes.

We have been able to obtain a plateau-like feature for $T_{\text {eff }}>5800 \mathrm{~K}$ only when the age of the sample is equal or higher than about 13.5 Gyr. Lower ages show a pronounced drop of [Li] at the TO which is not observed. Above this age, in the limit of the actual available observational samples and typical errors, the theoretical plateau region looks quite similar to the observational counterpart. In Fig. 4 we display the outcome of one simulation, where the age of the stars is randomly distributed between 13.5 and 14 Gyr. The result is quite typical of the ensemble of the 30 samples we have drawn with this age distribution. The plateau for $T_{\text {eff }}$ higher than about $5800 \mathrm{~K}$ (filled circles) is clearly visible together with a drop at lower $T_{\text {eff }}$ (open circles) due to the effect of pre-MS depletion. The solid line in the plane $[\mathrm{Li}]-T_{\text {eff }}$ shows the average $[\mathrm{Li}]$ value for these plateau objects $(\langle[\mathrm{Li}]\rangle)$, while the dashed lines indicate $\langle[\mathrm{Li}]\rangle \pm 3 \sigma$, where $\sigma$ is the typical observational error on $[\mathrm{Li}]$ adopted in our simulations (0.07 dex). Only 4 plateau stars appear to be outside the zone limited by the dashed lines (with lower [Li] values). If we take out these 4 stars and recompute $\langle[\mathrm{Li}]\rangle$, its value is not affected appreciably and all the remaining stars still lie within $3 \sigma$ of $\langle[\mathrm{Li}]\rangle$. For the stars within $3 \sigma$ of $\langle[\mathrm{Li}]\rangle$ there is no significant correlation between $[\mathrm{Li}]$ and $T_{\text {eff }}$. Plateau stars outside this region we will call "outliers". In all our simulations the outliers lie always below $\langle[\mathrm{Li}]\rangle-3 \sigma$, and they look like the theoretical counterpart of the observed Lidepleted stars in the plateau region (see Fig. 3; Thorburn 1994; Ryan et al. 2001). It is important to notice that in the observational analyses of the plateau, the (few) stars showing a substantial depletion with respect to the average plateau value, are not considered in the derivation of the plateau properties. As we did the same with our outliers, our selection closely mimicks the observational analyses.

By averaging over the 30 realizations with this same age distribution, we derived for the plateau stars a $[\mathrm{Li}]$ depletion by 0.27 dex and a derivative $\Delta[\mathrm{Li}] / \Delta[\mathrm{Fe} / \mathrm{H}]=$ 0.06 . The average number of outliers (Li-depleted stars) is 6. This latter number compares well with the actually observed number of Li-depleted stars in this same $[\mathrm{Fe} / \mathrm{H}]$ and $T_{\text {eff }}$ range (see Thorburn 1994). Also the average plateau value $[\mathrm{Li}]=2.23$, obtained from an initial lithium abundance $[\mathrm{Li}]_{\mathrm{o}}=2.50$, agrees well with the values provided in the literature, which span a relatively large range from $\sim 2.10$ (Ryan et al. 1999) up to $\sim 2.40$ (Gratton et al. 2000). The slope $\Delta[\mathrm{Li}] / \Delta[\mathrm{Fe} / \mathrm{H}]$ is halfway between the value $0.118 \pm 0.023$ claimed by Ryan et al. (1999), and the results by Bonifacio \& Molaro (1997), who did not find any dependence on $[\mathrm{Fe} / \mathrm{H}]$ for the plateau [Li] values.

The lower $T_{\text {eff }}$ limit off the plateau has been, somewhat arbitrarily, set to $5800 \mathrm{~K}$, which is a typical value as deduced from the observations. Had we increased it up to $5900 \mathrm{~K}$ (again, compatible with observations), the situation would have changed only slightly, with just a small decrease of the average [Li] depletion along the plateau, and a somewhat narrower distribution of the plateau stars around $\langle[\mathrm{Li}]\rangle$.

We made simulations also assuming a constant age of, respectively, 13.5 and $14.0 \mathrm{Gyr}$, and ages between 14 and $15 \mathrm{Gyr}$; the results are substantially the same, in the sense that a plateau-like feature is always found, without a significant depletion at its hotter end. We did not explore ages larger than about 15 Gyr because of the constraints put on the ages of field halo stars by the current estimates of globular clusters ages (see next section), and also by the fact that the horizontal extension of the plateau region is very much reduced and, even taking into account current uncertainties in the $T_{\text {eff }}$, it is too much reduced in comparison with observational data.

We now investigate whether our assumed metallicity distribution for halo stars leads to properties of the simulated sample in agreement with observed ones. To this end we compare in Fig. 5 the distribution of plateau stars 

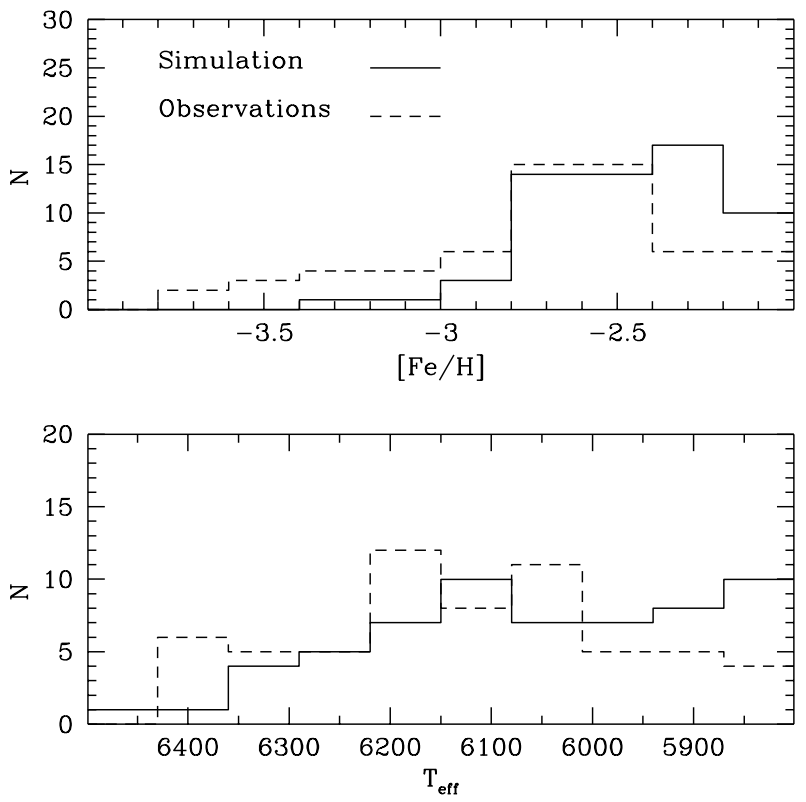

Fig. 5. Comparison of the number of plateau stars as a function of their $[\mathrm{Fe} / \mathrm{H}]$ (upper panel) and $T_{\text {eff }}$ (lower panel), between one of our MC samples and the observational data by Thorburn (1994) (see text for details).

$\left(T_{\text {eff }}>5800 \mathrm{~K}\right)$ as a function of the actual metallicity $[\mathrm{Fe} / \mathrm{H}]$ and $T_{\text {eff }}$ in case of one of our MC samples, and the data by Thorburn (1994) with $[\mathrm{Fe} / \mathrm{H}]<-2.0$ (the bin size is equal to $70 \mathrm{~K}$ in $T_{\text {eff }}$ and $0.20 \mathrm{dex}$ in $\left.[\mathrm{Fe} / \mathrm{H}]\right)$. The number of plateau stars in both cases is the same.

If one takes into account the spread of the empirical determinations of $T_{\text {eff }}$ and $[\mathrm{Fe} / \mathrm{H}]$ previously discussed the differences between the distributions of stars in the observed and synthetic samples are probably not very significant. However, as a test, we computed a series of MC simulations in the same way as before (ages again distributed uniformly between 13.5 and $14.0 \mathrm{Gyr}$ ), but considering an actual metallicity distribution biased towards lower metallicities with respect to the Ryan \& Norris (1991) one. This corresponds, basically, to have synthetic samples of plateau stars with a higher fraction of very metal-poor stars, in the assumptions that the observations were biased towards the most metal poor objects.

The distribution of plateau stars as a function of [Fe/H] and $T_{\text {eff }}$ for one of these latest simulations is shown in Fig. 6; the agreement between the two distributions is improved. The analysis of the [Li] abundances in these latest samples does not reveal any appreciable change with respect to the case of a standard halo metallicity distribution. This strengthens our conclusions, in the sense that they are not critically dependent on the actual $[\mathrm{Fe} / \mathrm{H}]$ distribution adopted in the simulations.

Before concluding this section, we would like to discuss briefly the spread of the plateau $\left(\sigma_{[\mathrm{Li}]}^{\mathrm{p}}\right)$ obtained in our simulations. If we consider all stars in the plateau region bar the outliers, we obtain a dispersion $\sigma_{[\mathrm{Li}]}^{\mathrm{p}}=0.09$. This number compares very well with the values of $\sigma_{[\mathrm{Li}]}^{\mathrm{p}}$
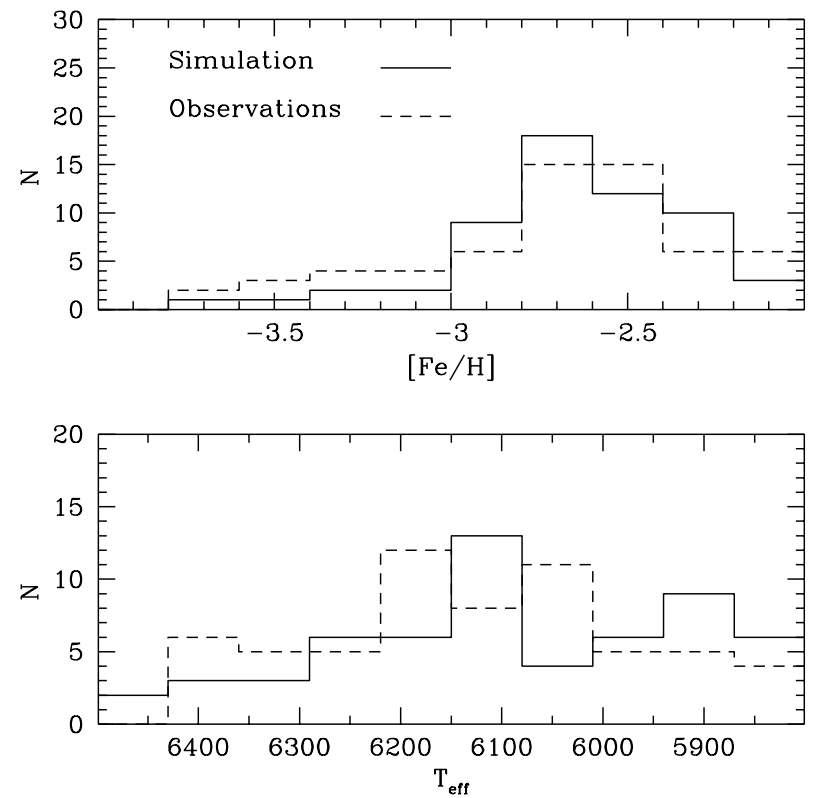

Fig. 6. As in the previous figure, but for a synthetic sample drawn from a metallicity distribution biased towards the most metal poor objects.

derived by, e.g., Spite et al. (1996) and Bonifacio \& Molaro (1997), for observational errors in [Li] similar to the ones used in our simulations. On the other hand, $\sigma_{[\mathrm{Li}]}^{\mathrm{p}}=0.09$ is definitely larger than the value given by Ryan et al. (1999). They have reobserved a sample of known plateau stars (thus avoiding contamination from Li-depleted objects), claiming average $1 \sigma$ errors by $\sim 30 \mathrm{~K}$ in the stellar temperatures, and by 0.033 dex in the derived [Li] values. They selected 22 stars around the TO of the field population, with $T_{\text {eff }}$ between $6100 \pm 50 \mathrm{~K}$ and $6300 \pm 50 \mathrm{~K}$, $[\mathrm{Fe} / \mathrm{H}]$ between -2.5 and -3.5 , and found that the $1 \sigma$ dispersion of [Li] around an average value of 2.11 is equal to 0.053 dex (they went on discarding 3 stars more discrepant from the average to obtain an even smaller dispersion).

We performed a series of MC simulations by drawing samples of 25 stars among the samples of plateau stars obtained from the previous simulations. We selected the stars in the same $[\mathrm{Fe} / \mathrm{H}]$ range as Ryan et al. (1999); under the hypothesis that their hottest stars were stars at the TO, and for avoiding problems related to zero-point uncertainties in the temperature scale, we restricted our sample to stars with $T_{\text {eff }}$ ranging between the hottest value reached in our simulations and a value $200 \mathrm{~K}$ lower. The extremes of this $T_{\text {eff }}$ range are similar to the values adopted by Ryan et al. (1999). By averaging over 30 of these realizations we obtained an average $[\mathrm{Li}]=2.17$, with a $1 \sigma$ dispersion of 0.075 dex. This average [Li] value is lower than the value derived previously; this is easily explained by the derivative $\Delta[\mathrm{Li}] / \Delta[\mathrm{Fe} / \mathrm{H}]=0.06$ obtained before, and the fact that in these latter simulations we are considering only $[\mathrm{Fe} / \mathrm{H}] \leq-2.5$. What is probably relevant is that the spread we find is larger than the value obtained by Ryan et al. (1999), but we would like to recall the results by Asplund et al. (1999) which clearly raise 


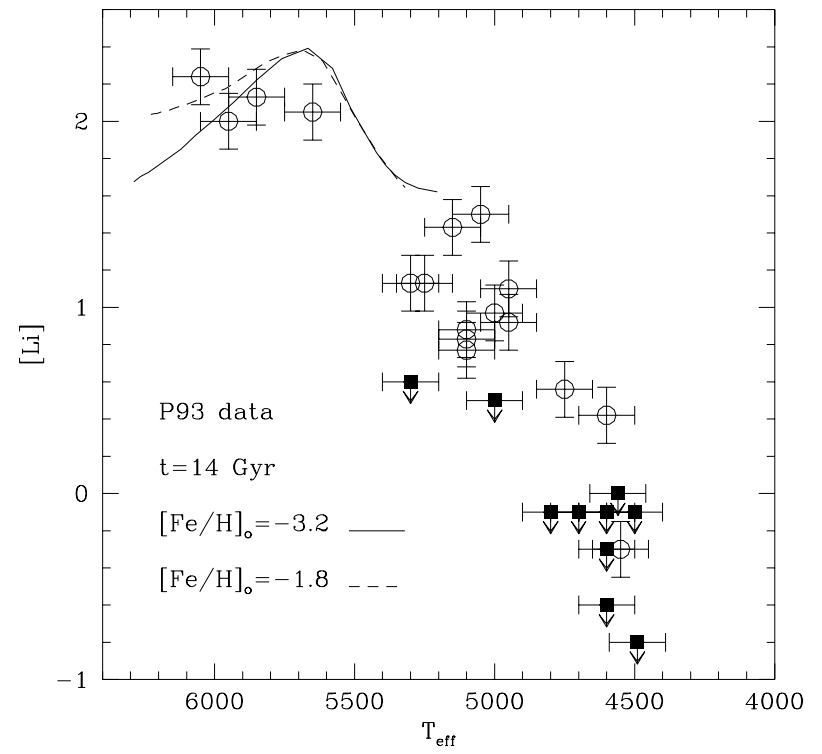

Fig. 7. Comparison in the $[\mathrm{Li}]-T_{\text {eff }}$ plane, between the field subgiant data by Pilachowski et al. (1993) for $[\mathrm{Fe} / \mathrm{H}] \leq-2.0$, and our isochrones with $t=14$ Gyr for two different initial metallicities. Squares correspond to upper limits.

the possibility that current determinations of the plateau dispersion (and this would hold also for the larger samples discussed earlier) are underestimated appreciably.

\subsection{Field subgiant branch stars}

If one accepts, as a working hypothesis, an age between 13.5 and 14 Gyr for the most metal-poor field stars, it is necessary to see if this value satisfies constraints coming from Li abundances in the SGB phase.

We compare our models with observational data by Pilachowski et al. (1993) for metallicities lower than $[\mathrm{Fe} / \mathrm{H}]=-2$. In Fig. 7 we show the comparison of the data with the SGB part of our isochrones, for an initial $[\mathrm{Fe} / \mathrm{H}]_{\mathrm{o}}$ equal to -3.2 (solid line) and -1.8 (dashed line), and $t=14$ Gyr. Because of the extremely low number of data points on the SGB and the size of their error bars, we simply compared the position of the observational data with SGB isochrones bracketing the metallicity range spanned by the observations. Keeping in mind possible systematic errors in the $T_{\text {eff }}$ scale (which translates also into systematic errors in $[\mathrm{Li}]$ ), with respect to the data for the MS, it is interesting to notice that the models with diffusion together with our assumed initial $[\mathrm{Li}]_{\mathrm{o}}=2.50$, are not in contradiction with observations. However, the size of the observed sample and of the error bars does not permit to definitely assess the presence (or absence) of the peak in the [Li] abundance predicted by models with diffusion.

The steep decrease of $[\mathrm{Li}]$ for decreasing $T_{\text {eff }}$ (equivalently: for increasing luminosity) along the RGB is neither reproduced by models with diffusion, nor by standard models (see the discussion in Pilachowski et al. 1993). At temperatures lower than the endpoint of the isochrones shown in Fig. 7, [Li] would stay constant, since the

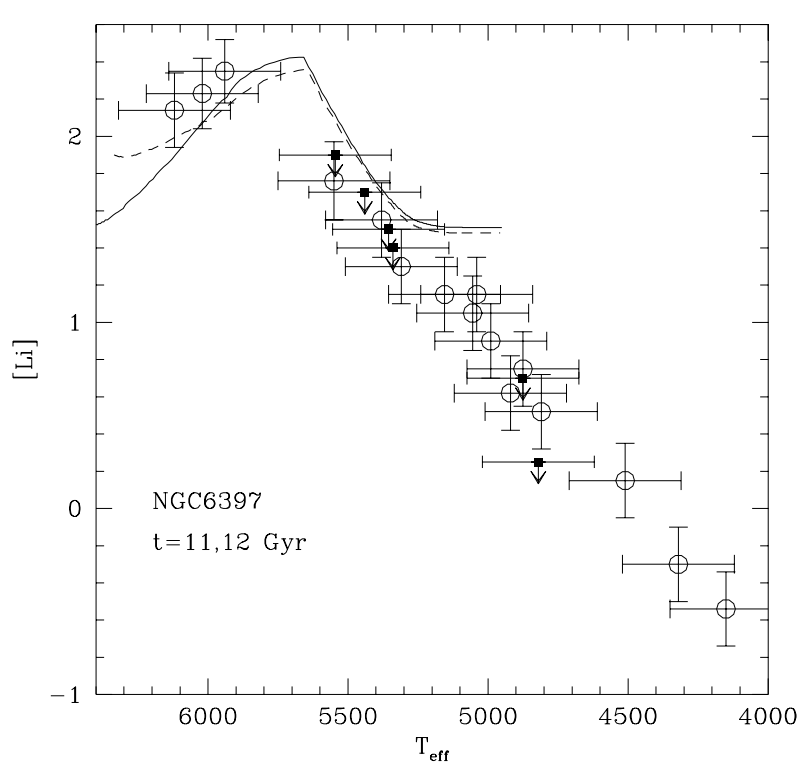

Fig. 8. Run of $[\mathrm{Li}]$ as a function of $T_{\text {eff }}$ for SGB and base of the RGB stars in the globular clusters NGC 6397. Symbols are as in Fig. 7.

convective envelope has reached its maximum extension and starts now to recede slowly due to the outward movement of the H-burning shell. The further depletion can be explained only by invoking extra deep mixing (see, e.g., Kraft 1994; Denissenkov \& Weiss 1996; Weiss et al. 2000).

\subsection{Globular cluster subgiant branch stars}

Figure 8 shows a comparison with Li abundances in the globular cluster NGC6397 SGB stars. In a globular cluster, all stars have the same initial chemical composition and the same age; we adopted an initial $[\mathrm{Fe} / \mathrm{H}]_{\mathrm{o}}$ equal to -1.8, following the results by Carretta \& Gratton (1997) for RGB stars belonging to this cluster. This choice is justified by the fact that at the photosphere of RGB stars the metallicity is restored to the initial value due to the deepening of the convective envelope, which engulfs almost all the metals diffused towards the core during the MS phase (Fig. 2). Ages of 11 and 12 Gyr have been employed, according to the results by Salaris \& Weiss (1998). It is worth noticing that in this case the constraint on the stellar ages comes from a method completely unrelated to the $\mathrm{Li}$ abundance determinations. This age is lower than the age of the halo field stars previously derived from the Li-plateau, but in any case an increase of NGC 6397 age by 1-2 Gyr would not alter substantially the result of this comparison.

The observational data are from Pasquini \& Molaro (1996) for SGB stars and Castilho et al. (2000) for RGB stars. The $T_{\text {eff }}$ values by Pasquini \& Molaro (1996) have been reduced by $60 \mathrm{~K}$ to take into account the fact that their assumed reddening was 0.01 mag larger than the one used by Castilho et al. (2000); the corresponding [Li] 
values consequently have been reduced according to the derivative $\Delta[\mathrm{Li}] / \Delta T_{\text {eff }}$ provided in the mentioned papers.

Due to the small sample of SGB stars observed, their comparison with models including diffusion does not permit - like in the case of field SGB stars - to draw definitive conclusions about the efficiency of this process in globular clusters stars. An analogous conclusion is reached comparing our models with the small sample of 6 SGB stars in the cluster M 92 observed by Boesgaard et al. (1998). Notice, again, the problem along the RGB - very similar to the case of field stars - where models (standard and diffusive ones) cannot reproduce the trend of [Li] with repect to $T_{\text {eff }}$.

\subsection{Field stars with $-2.0<[\mathrm{Fe} / \mathrm{H}]<-1.5$}

Up to now we concentrated on the low-metallicity end of the Spite-plateau, which, however extends also to higher $[\mathrm{Fe} / \mathrm{H}]$. We now consider stars with $-2.0<[\mathrm{Fe} / \mathrm{H}]<-1.5$, in order to see if a plateau can be found also when including this metallicity range. We computed a series of models and Li-isochrones with initial iron content $[\mathrm{Fe} / \mathrm{H}]_{\mathrm{o}}=-1.3$ $(Z=0.002)$ and then performed MC simulations selecting this time a sample of $\sim 35$ plateau stars with $-2.0<[\mathrm{Fe} / \mathrm{H}]<-1.5$ (which is approximately the size of the observed sample in this metallicity range). In Fig. 9 we plot (filled circles) the result of one simulation (only plateau stars), together with the result for the stars with $[\mathrm{Fe} / \mathrm{H}] \leq-2.0$ (open circles) from Fig. 4. The solid and dashed lines have the same meaning as in Fig. 4. For the more metal-rich stars we have assumed an age randomly distributed between 12.5 and 13 Gyr, to simulate a decrease of the average age of Pop II field stars for increasing metallicity, above $[\mathrm{Fe} / \mathrm{H}]=-2.0$. The average value of [Li] is still 2.23 , as in the case of the sample including only stars with $[\mathrm{Fe} / \mathrm{H}] \leq-2.0$. The selected age does not play a decisive role. Variations of the order of \pm 1 Gyr do not affect appreciably the overall picture.

We have also compared the Li abundances from our models with the observations by Pilachowski et al. (1993) for more metal-rich SGB and RGB stars of $-2.0<$ $[\mathrm{Fe} / \mathrm{H}]<-1.5$. There appears no inconsistency between theory and observations besides the fact that also in this metallicity range there is the well-known problem of an additional depletion observed in RGB stars. The Li-peak predicted around $5600 \mathrm{~K}$ might be visible, but given the scarce data and rather large errors cannot be claimed to exist, just as in the case of the very metal-poor field stars.

\section{Discussion and conclusions}

In the previous section we have compared our stellar models including uninhibited atomic diffusion with observations of $[\mathrm{Li}]$ in metal-poor $([\mathrm{Fe} / \mathrm{H}] \leq-2.0)$ halo field and globular clusters stars. We have shown that there is no apparent contradiction with observations, at least with the present observational data, as long as the age of field subdwarfs is of the order of $13.5 \mathrm{Gyr}$ or more when

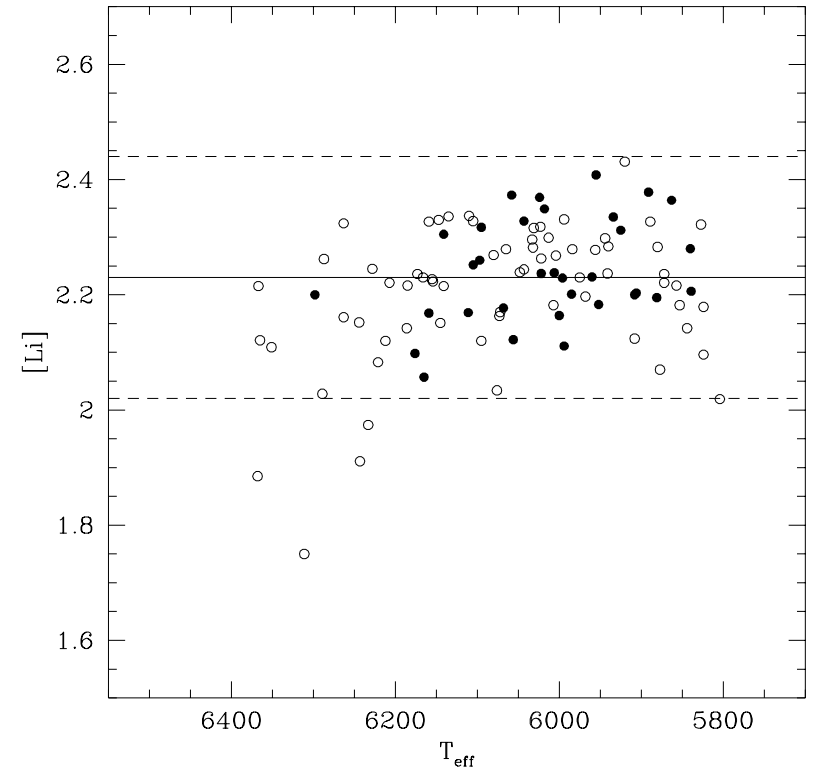

Fig. 9. Run of [Li] as a function of $T_{\text {eff }}$ for stars in one of our MC simulations (see text for details); open circles are stars with $[\mathrm{Fe} / \mathrm{H}] \leq-2.0$, while filled circles are stars with $-2.0<$ $[\mathrm{Fe} / \mathrm{H}]<-1.5$.

$[\mathrm{Fe} / \mathrm{H}] \leq-2.0$. This is, of course, not a proof that diffusion is fully efficient in halo stars (since in general also standard isochrones can explain the observations; e.g., Vauclair \& Charbonnel 1995); however, our analysis shows that it is not justified to discard models with fully efficient diffusion on the base of current measurements of [Li] in metal-poor stars.

In particular, the Li-plateau region is satisfactorily reproduced by our MC simulations, for the ages discussed before. This is at odds with conclusions from previous analyses which were based mainly on the comparison of an isochrone of a given age and a single value of $[\mathrm{Fe} / \mathrm{H}]$ with the moderately small observational samples of stars with different metallicities and possibly different ages. In our MC simulations we could take into account the actual number of stars with [Li] measurements, their observed metallicity distribution and the typical observational errors. The fact that we do not find in the simulations a clear depletion of $[\mathrm{Li}]$ at the hotter end of the plateau is only partly due to the ages we selected for the stars with $[\mathrm{Fe} / \mathrm{H}]<-2.0$. In fact, lower ages (see Fig. 1) produce a more visible depletion at the TO, but still, even for ages of about 14 Gyr and taking into account the observational errors, when $[\mathrm{Fe} / \mathrm{H}] \leq-2.6$, a drop at the highest end of the plateau has to be visible, provided that a sufficiently high number of stars is observed. From our simulations we derive that $[\mathrm{Li}]$ measurements of a sample of about 200 plateau stars (about 40-50 of them at the hottest end of the plateau) with $[\mathrm{Fe} / \mathrm{H}] \leq-2.6$ are needed in order to clearly detect this drop, keeping unchanged the observational errors (see Fig. 10).

For the more metal-rich objects of Sect. 3.4 with $[\mathrm{Fe} / \mathrm{H}]$ above -2.0 an increase of the observed sample size would 


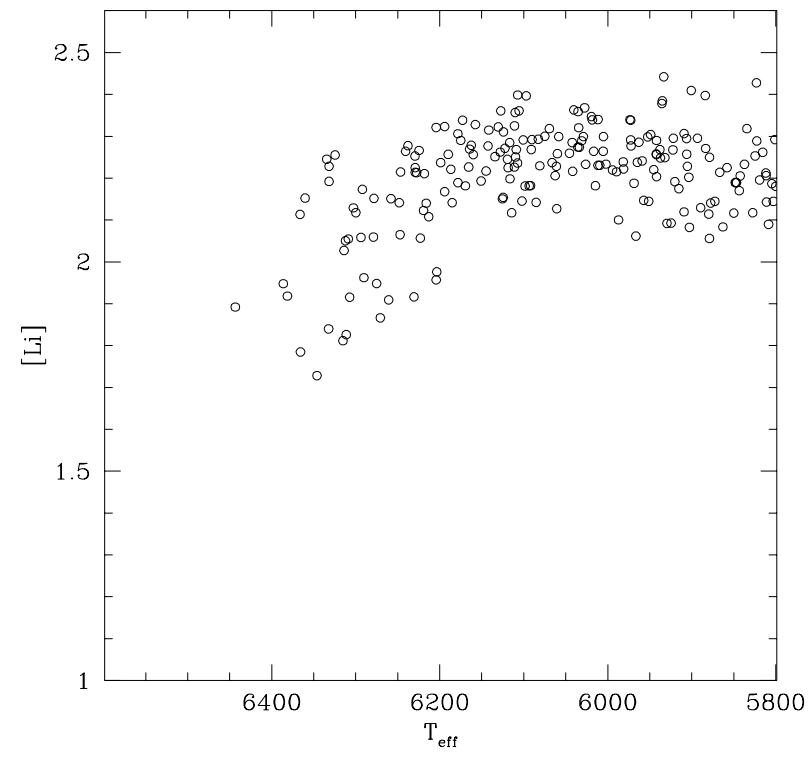

Fig. 10. Results of a MC simulation with $\sim 200$ plateau stars and $[\mathrm{Fe} / \mathrm{H}] \leq-2.6$ (see text for details). The drop in $[\mathrm{Li}]$ at the TO due to diffusion is now clearly apparent.

not change the plateau morphology, since at these metallicities there is no abrupt drop of [Li] at the TO region, but an almost constant value of $[\mathrm{Li}]$ all along the plateau region.

It has been mentioned several times in the literature that ${ }^{6} \mathrm{Li}$, which is destroyed by proton-captures at even lower temperatures than ${ }^{7} \mathrm{Li}\left(2 \times 10^{6} \mathrm{~K}\right)$, would be an additional and strong indicator of ${ }^{7} \mathrm{Li}$ destruction (see, for example, Vangioni-Flam et al. 1999). What observations provide is the sum ${ }^{7} \mathrm{Li}+{ }^{6} \mathrm{Li}$. Disentangling the abundance of one isotope from the other one is a very difficult task; it is based on fitting the observed profile of the Li I blend with theoretical profiles calculated from model stellar atmospheres characterized by different ${ }^{6} \mathrm{Li} /{ }^{7} \mathrm{Li}$ ratios (see, e.g., Hobbs et al. 1999). Until now all (very few) measured Li-isotope ratios (e.g. Nissen et al. 1999; Hobbs et al. 1999; Nissen et al. 2000) are either upper limits only or are so high $\left({ }^{6} \mathrm{Li} /\left({ }^{7} \mathrm{Li}+{ }^{6} \mathrm{Li}\right) \approx 0.05\right)$ that they exceed limits from Big Bang Nucleosynthesis by orders of magnitude, such that galactic chemical evolution (Vangioni-Flam et al. 1999) has to be taken into account to explain them. From this point on, of course, all conclusions about stellar depletion of lithium isotopes are subject to the acceptance of these chemical evolution models. The quoted papers arrive at conclusions both excluding (Vangioni-Flam et al. 1999) and requiring stellar depletion (Hobbs et al. 1999). We therefore conclude that presently ${ }^{6} \mathrm{Li}$ does not provide additional conclusive information about diffusive processes in low-mass stars. We note that along the MS diffusion alone would only mildly change the isotope ratio, anyway, with an increase of the initial ${ }^{6} \mathrm{Li} /\left({ }^{7} \mathrm{Li}+{ }^{6} \mathrm{Li}\right)$ value by at most $40 \%$.

In principle, useful constraints on diffusion in low-mass stars could come from spectroscopy along globular cluster CMDs, as the one shown in Fig. 8 for NGC 6397. Recently,
Gratton et al. (2001) have presented VLT/UVES results about element abundances in stars at the TO and at the RGB base in the same cluster and in NGC 6752. In NGC 6397 rather constant abundance values are found, and in particular $[\mathrm{Fe} / \mathrm{H}]$ appears to be exactly the same in both groups of stars, a fact which would strongly disfavour diffusion. Also Li, which is not discussed in this paper, shows very small star-to-star variations (Gratton, private communication). Although comparable work (Carretta \& Gratton 1997; Castilho et al. 2000) returned somewhat different results concerning the Fe-abundance, Gratton et al. (2001) certainly have presented a strong case against the efficiency of diffusion in NGC 6397.

On the contrary in NGC 6752 , even if $[\mathrm{Fe} / \mathrm{H}]$ appears to be the same in TO and SGB stars, large star-to-star scatter in element abundances is present within stars of the same evolutionary stage, indicating additional stellar processes at work. This applies also to lithium (Gratton, private communication). It therefore appears that clusterto-cluster differences may be present and much more work along the line of Gratton et al. (2001) - in particular for the lowest metallicity clusters, where the effect of diffusion, if present, should be larger - is needed to clarify this. It might also turn out that there are systematic differences between the efficiency of diffusion in clusters and field stars, just as in the case of RGB abundance anomalies (Gratton et al. 2000).

Assuming that diffusion indeed has taken place in field Spite-plateau stars, our results have significant bearings for the question concerning the cosmological baryon density. We reproduce the observed Spite-plateau [Li]abundance when assuming a primordial value of $[\mathrm{Li}]=2.5$. According to standard Big Bang Nucleosynthesis (see, for example, the recent calculations by Vangioni-Flam et al. 2000), this corresponds to a baryon-to-photon ratio $\eta$ of $5.1 \times 10^{-10}$ (see Fig. 4 of the quoted paper), which is in almost perfect agreement with the low deuterium value $\left(\mathrm{D} / \mathrm{H}=3 \ldots 4 \times 10^{-5}\right)$ by Tytler et al. (2000) and the high ${ }^{4} \mathrm{He}$ abundance of 0.244 by Izotov \& Thuan (1998). Since $\eta$ is related to the baryonic density via $\eta=273 \times 10^{-10} \Omega_{\mathrm{B}} h^{2}$ (with $h$ being the Hubble constant in units of $\left.100 \mathrm{kms}^{-1} \mathrm{Mpc}^{-1}\right)$, this results in $\Omega_{\mathrm{B}}=0.019 h^{-2}$. Taking $h=0.71$ (Mould et al. 2000) yields $\Omega_{\mathrm{B}}=0.038$. This result is within $1 \sigma$ of the value inferred from the BOOMERANG experiment (Netterfield et al. 2001), which is $\Omega_{\mathrm{B}} h^{2}=0.022 \pm 0.003$.

Our derived cosmological baryon density is obtained under the assumption of no significant galactic production of ${ }^{7} \mathrm{Li} .{ }^{6} \mathrm{Li}$ measurements can in principle be used also to put useful constraints on the amount of ${ }^{7} \mathrm{Li}$ produced by galactic chemical evolution, but the available measurements (see also the previous discussion) do not provide yet a consistent picture of the abundance ratio of these two isotopes. As an example, the value ${ }^{6} \mathrm{Li} /\left({ }^{7} \mathrm{Li}+{ }^{6} \mathrm{Li}\right) \approx 0.05$ for the metal poor TO star HD $84937([\mathrm{Fe} / \mathrm{H}] \approx-2.3)$ points to a non-negligible contribution of ${ }^{6} \mathrm{Li}$ - and therefore also ${ }^{7} \mathrm{Li}$ - from cosmic ray production. However, measurements of the same quantity for HD 218502, a star with 
similar metallicity and evolutionary status, provide an upper limit of only 0.02 and a best fit value equal to zero (see Hobbs et al. 1999), consistent with no contribution from galactic chemical evolution. If in the future ${ }^{6} \mathrm{Li}$ measurements will consistently indicate a significant influence of galactic production of ${ }^{7} \mathrm{Li}$ (of order 0.1 dex or more), one will have to reconsider our conclusions about the cosmological baryon density.

One could in principle worry about the fact that our adopted initial $[\mathrm{Li}]$ abundance implies a primordial $Y \approx$ 0.24 , while in our models we used $Y=0.23$. However, as we have verified with appropriate computations, the effect of using $Y=0.24$ as the initial He abundance for our models is just an age shift. More in detail, the [Li] abundance as a function of $T_{\text {eff }}$ along an isochrone of fixed initial $[\mathrm{Fe} / \mathrm{H}]_{\mathrm{o}}$ and age computed with an initial $Y=0.23$ would be equal to the abundances of an isochrone with the same initial $[\mathrm{Fe} / \mathrm{H}]_{\mathrm{o}}$ but an age lower by $1 \mathrm{Gyr}$, if an initial $Y=0.24$ is used. The same holds for the $[\mathrm{Fe} / \mathrm{H}]$ values along an isochrone. Our conclusions about the Li-plateau are therefore not affected at all, apart from the fact that in this case the minimum age required to reproduce the observed plateau would be 12.5 Gyr.

We finally mention that our treatment of diffusion does not take into account the effect of radiative levitation. The diffusive velocities by Thoul et al. (1994), which we are using, might be overestimated by $25 \%$ on average, at least in the case of the Sun, according to Turcotte et al. (1998). It is plausible, but not certain, that a similar overestimate might happen for metal-poor stars. We neither included mass loss in the calculations, which is also known to reduce the effect of diffusion. For mass loss rates compatible with current observations the surface abundances depletion is not affected appreciably, but strong stellar wind on the MS may inhibit diffusion completely (Vauclair \& Charbonnel 1995). Due to the neglect of both radiative levitation and mass loss, our models probably overestimate the depletion to some degree, even if we cannot quantify this up to now.

We can conclude that the Spite plateau, given present day observational sample sizes, can be reproduced by models including atomic diffusion and is therefore (alone) no strong argument against the presence of sedimentation in low-mass metal-poor field stars.

Acknowledgements. A.W. is grateful for the hospitality at the Institute for Advanced Study and Princeton Observatory and for a Fulbright fellowship which allowed visiting both places. Helpful discussions with J. Truran, L. M. Hobbs, R. Cayrel, C. Deliyannis, K. Jedamzik, and F. D'Antona are acknowledged. We thank an anonymous referee for his/her comments, which helped to improve the quality of the paper.

\section{References}

Angulo, C., Arnould, M., Rayet, M., et al. 1999, Nucl. Phys. A, 656, 3

Bahcall, J. N., Pinsonneault, M. H., Basu, S., \& ChristensenDalsgaard, J. 1997, Phys. Rev. Lett., 78, 171

Bonifacio, P., \& Molaro, P. 1997, MNRAS, 285, 847
Brun, A. S., Turck-Chiéze, S., \& Zahn, J. P. 1999, ApJ, 525, 1032

Carretta, E., \& Gratton, R. G. 1997, A\&AS, 121, 95

Cassisi, S., Castellani, V., Degl'Innocenti, S., \& Weiss, A. 1998, A\&AS, 129, 267

Castellani, V., Ciacio, F., Degl'Innocenti, S., \& Fiorentini, G. 1997, A\&A, 322, 801

Castellani, V., \& Degl'Innocenti, S. 1999, A\&A, 344, 97

Castilho, B. V., Pasquini, L., Allen, D. M., Barbuy, B., \& Molaro, P. 2000, A\&A, 361, 92

Chaboyer, B., Deliyannis, C. P., Demarque, P., Pinsonneault, M. H., \& Sarajedini, A. 1992, ApJ, 388, 372

Chaboyer, B., Demarque, P., \& Pinsonneault, M. H. 1995, ApJ, 441,865

D'Antona, F., \& Mazzitelli, I. 1984, A\&A, 138, 431

Deliyannis, C. P., \& Demarque, P. 1991, ApJ, 379, 216

Deliyannis, C. P., Demarque, P., \& Kawaler, S. D. 1990, ApJS, 73,21

Denissenkov, P. A. \& Weiss, A. 1996, A\&A, 308, 773

Gratton, R. G., Sneden, C., Carretta, E., \& Bragaglia, A. 2000, A\&A, 354, 169

Gustafsson, B., Bell, R. A., Eriksson, K., \& Nordlund, A. 1975, A\&A, 42, 407

Hobbs, L. M., Thorburn, J. A., \& Rebull, L. M. 1999, ApJ, 523,797

Kraft, R. P. 1994, PASP, 106, 553

Kurucz, R. L. 1993, ATLAS9 Stellar Atmosphere Programs, CD-ROM 13

Morel, P., \& Baglin, A. 1999, A\&A, 345, 156

Mould, J. R., Huchra, J. P., Freedman, W. L., et al. 2000, ApJ, 529,786

Netterfield, C. B., Ade, R. A. R., Bock, J. J., et al. 2001, preprint [astro-ph/0104460]

Nissen, P., Lambert, D., Primas, F., \& Smith, V. 1999, A\&A, 348,211

Nissen, P. E., Asplund, M., Hill, V., \& D'Odorico, S. 2000, A\&A, 357, L49

Pilachowski, C. A., Sneden, C., \& Booth, J. 1993, ApJ, 407, 699

Richard, O., Vauclair, S., Charbonnel, C., \& Dziembowski, W. A. 1996, A\&A, 312, 1000

Ryan, S. G., Beers, T. C., Deliyannis, C. P., \& Thorburn, J. A. 1996, ApJ, 458, 543

Ryan, S. G., Kajino, T., Beers, T. C., et al. 2001, ApJ, 549, 55

Ryan, S. G., Norris, J. E., \& Beers, T. C. 1999, ApJ, 523, 654

Salaris, M., Degl'Innocenti, S., \& Weiss, A. 1997, ApJ, 479, 665

Salaris, M., Groenewegen, M. A. T., \& Weiss, A. 2000, A\&A, 355,299

Salaris, M., \& Weiss, A. 1998, A\&A, 335, 943

Spite, F., \& Spite, M. 1982, A\&A, 115, 357

Spite, M., Francois, P., Nissen, P. E., \& Spite, F. 1996, A\&A, 307,172

Thorburn, J. A. 1994, ApJ, 421, 318

Vangioni-Flam, E., Cassé, M., Cayrel, R., et al. 1999, New Astron., 4, 245

Vangioni-Flam, E., Coc, A., \& Cassé, M. 2000, A\&A, 360, 15

Vauclair, S. 1999, A\&A, 351, 973

Vauclair, S., \& Charbonnel, C. 1995, A\&A, 295, 715

Vauclair, S., \& Charbonnel, C. 1998, ApJ, 502, 372

Vazdekis, A., Salaris, M., Arimoto, N., \& Rose, J. A. 2001, ApJ, 549, 274

Weiss, A., Denissenkov, P. A., \& Charbonnel, C. 2000, A\&A, 356,181

Weiss, A., \& Schlattl, H. 2000, A\&AS, 144, 487 